\title{
Crecimiento del pez ornamental Hemichromis bimaculatus (Cichlidae) en un Sistema de Recirculación de Agua, con incorporación en el alimento de residuos industriales y algas
}

Growth of the ornamental fish Hemichromis bimaculatus (Cichlidae) in a Water Recirculation System, incorporating industrial residues and algae into the feed

\section{María Marta Pérsico}

Facultad Regional Mar del Plata, Universidad Tecnológica Nacional - Argentina mmartapersico@gmail.com

\section{Federico Alcides Cecchi}

Facultad Regional Mar del Plata, Universidad Tecnológica Nacional - Argentina federicocecchi@gmail.com (correspondencia)

\section{Aldo Nahuel Zanazzi}

Facultad Regional Mar del Plata, Universidad Tecnológica Nacional - Argentina nahuelzanazzi@gmail.com

\section{Arturo Asiain}

Facultad Regional Mar del Plata, Universidad Tecnológica Nacional - Argentina asiain164@gmail.com

\section{Brian Nahuel Tomaselli}

Facultad Regional Mar del Plata, Universidad Tecnológica Nacional - Argentina briannahueltomaselli@gmail.com

\section{Fabiana Pérez}

Facultad Regional Mar del Plata, Universidad Tecnológica Nacional - Argentina fabianadenajle@gmail.com

\section{María Inés Prario}

Facultad Regional Mar del Plata, Universidad Tecnológica Nacional - Argentina mariaprario@gmail.com

\section{Paula Waldmann}

Facultad Regional Mar del Plata, Universidad Tecnológica Nacional - Argentina paulawaldmann94@gmail.com

\section{Juan Carlos Mallo}

Facultad Regional Mar del Plata, Universidad Tecnológica Nacional - Argentina juancmallo@gmail.com

\section{María Laura Patat}




\title{
Resumen
}

El presente trabajo tuvo como objetivo evaluar el crecimiento de juveniles del pez ornamental $H$. bimaculatus, Cichlidae, utilizando residuos industriales de langostino argentino y cebada cervecera, con complemento de algas en el alimento. El cultivo se realizó en un sistema de recirculación de agua a escala de laboratorio. Se evaluaron el peso y largo total de los individuos, la supervivencia, su aspecto externo y la calidad del agua del sistema de cultivo. Se hallaron diferencias significativas $(p<0,05)$ entre las medianas de peso y largo total al compararlas con el tratamiento control, con 47,5 \% de proteínas, con resultados más favorables respecto al experimental; en éste la tasa de crecimiento específico fue $2 \mathrm{mg} /$ día y la sobrevivencia $98 \%$. Los residuos de langostino y cebada podrían ser utilizados como ingredientes en alimentos formulados para esta especie, reemplazando parcialmente a la harina de pescado, de forma más amigable con el ambiente y alta disponibilidad regional.

Palabras clave: peces ornamentales, sistema de recirculación, residuos langostino y cebada cervecera, algas

\begin{abstract}
The objective of this study was evaluate the growth of juveniles of the freshwater ornamental fish $H$. bimaculatus, Cichlidae, using industrial waste of argentine prawn and beer barley, with algae supplements in the experimental food. The culture was carried out in a laboratory scale water recirculation system. The weight and total length of the individuals, survival, its external appearance and the water quality of the crop system were evaluated. Significant differences $(p<0.05)$ were found between the medians of weight and total length when compared with the control treatment, with $47.5 \%$ of proteins, with more favorable results compared to the experimental; in this the specific growth rate was $2 \mathrm{mg} /$ day and survival $98 \%$. Shrimp and barley residues could be used as ingredients in foods formulated for this species, partially replacing fishmeal, in a more environmentally friendly and regionally available manner.
\end{abstract}

Key words: ornamental fish, recirculation system, shrimp and beer barley residues, algae

\section{Introducción}

Los peces en el ambiente natural encuentran disponible diversos alimentos que satisfacen sus necesidades nutricionales y obtienen de él una dieta balanceada. En actividades de Acuicultura referidas particularmente a la cría de peces ornamentales deben asegurar, además de un buen desarrollo y crecimiento, un aspecto externo adecuado conforme a las características de cada especie que le asigna el valor individual.

Las dietas comerciales contienen los ingredientes necesarios según los requerimientos de la especie o etapa productiva específica (Hepher, 1993). Hoy las raciones empleadas en la acuicultura, en general, dependen para su suministro proteico de las pesqueras y de la agricultura, debido a que basan su producción en las harinas de pescado y de soja (Lam Romero et al, 2012). Estos factores, así como el costo elevado del alimento, exigen la búsqueda de fuentes alternativas de proteínas (Vásquez-Torres et al, 2011). Además, los pigmentos, aunque no son nutrientes, son considerados de gran importancia en las dietas para peces ornamentales, debido a que resaltan e intensifican los colores generando un aumento en el 
valor de venta y la aceptación para su comercialización. El mercado mundial de la astaxantina en la actualidad, está cubierto en gran medida por pigmentos sintéticos de elevado costo.

Entre las fuentes alternativas de proteínas para uso en acuicultura, la "cabeza de camarón” puede ser una opción económicamente recomendable en alimentos formulados para especies acuáticas, ya que ofrece diversas ventajas tales como bajos costos para su obtención y transformación a harina (Shoemaker y Richards-Rajaduraí, 1991; Cira et al, 2002; Honorato et al, 2006), un perfil de aminoácidos comparable con la harina de pescado, contenido de sustancias quimio-atractantes (Shoemaker y Richards-Rajaduraí, 1991; Hertrampf y Piedad-Pascual, 2000), y fuente natural de pigmentos carotenoides y quitina (Fox et al, 1994), no compitiendo con alimentos para el hombre. La astaxantina es un pigmento carotenoide presente en los caparazones de camarones, los cuales representan alrededor de un $40 \%$ del peso total en el langostino argentino (Pleoticus muelleri) y son desechos pesqueros de gran volumen en la zona patagónica de nuestro país (Lladser et al, 2014).

Asimismo, el bagazo de la cebada cervecera (Hordeum distichum) obtenido de la producción de cerveza artesanal, es un producto residual que proviene del macerado de los granos en agua para obtener azúcares, y que puede contener alrededor de un $20 \%$ de proteína bruta (Ferrari et al, 2017); esa actividad se encuentra muy desarrollada a nivel local.

Como complementos nutricionales varias especies de algas son utilizadas en alimentos para animales y en peces de ornato se incorporan en sus dietas comerciales. La microalga conocida comercialmente como Espirulina favorece el crecimiento, mejora la pigmentación y produce un aumento de la respuesta del sistema inmune; entre el 60-70 \% de su peso seco son proteínas, con un alto espectro de aminoácidos esenciales y, por ello, es de alto valor biológico. Así, la especie Arthrospira platensis se utiliza en dietas de peces ornamentales, por su efecto favorable en el aumento de la intensidad del color y con una inclusión de entre 1-5 \% en el alimento. En Argentina varias empresas la cultivan y producen a pequeña y mediana escala, en forma de cápsulas y harina (Argento et al, 2016). Por otra parte, la macroalga marina Undaria pinnatifida (Phaeophyceae) conocida vulgarmente como wakame, contiene proteínas entre 10 y $20 \%$, vitaminas, pigmentos carotenoides como fucoxantina y B-caroteno, fibras, y minerales como K, Na, Ca y Mg (Casas et al, 2010). La misma ha sido introducida accidentalmente en la costa patagónica y hoy cubre un área de costa de $100 \mathrm{~km}$ entre Puerto Deseado y Mar del Plata. Comercialmente se extrae por pesca artesanal o se colecta de arribazones, y se vende seca para utilizarse en productos elaborados o como condimento. Se ha incorporado, como otras especies de macroalgas, en alimentos comerciales importados de peces ornamentales.

El presente trabajo tuvo como objetivo evaluar el crecimiento de juveniles del pez ornamental de agua dulce $H$. bimaculatus, incorporando en el alimento harinas de residuos del langostino argentino y de bagazo de cebada, y complementos de harinas de algas Espirulina y Wakame. Para ello, se utilizó un Sistema de Recirculación de Agua (SRA) a escala de laboratorio.

\section{Materiales y Métodos}

El presente trabajo experimental se desarrolló en un sector del Laboratorio de Acuicultura, LACUI, Facultad Regional Mar del Plata (FRMdP), Universidad Tecnológica Nacional (Figura $1)$. 


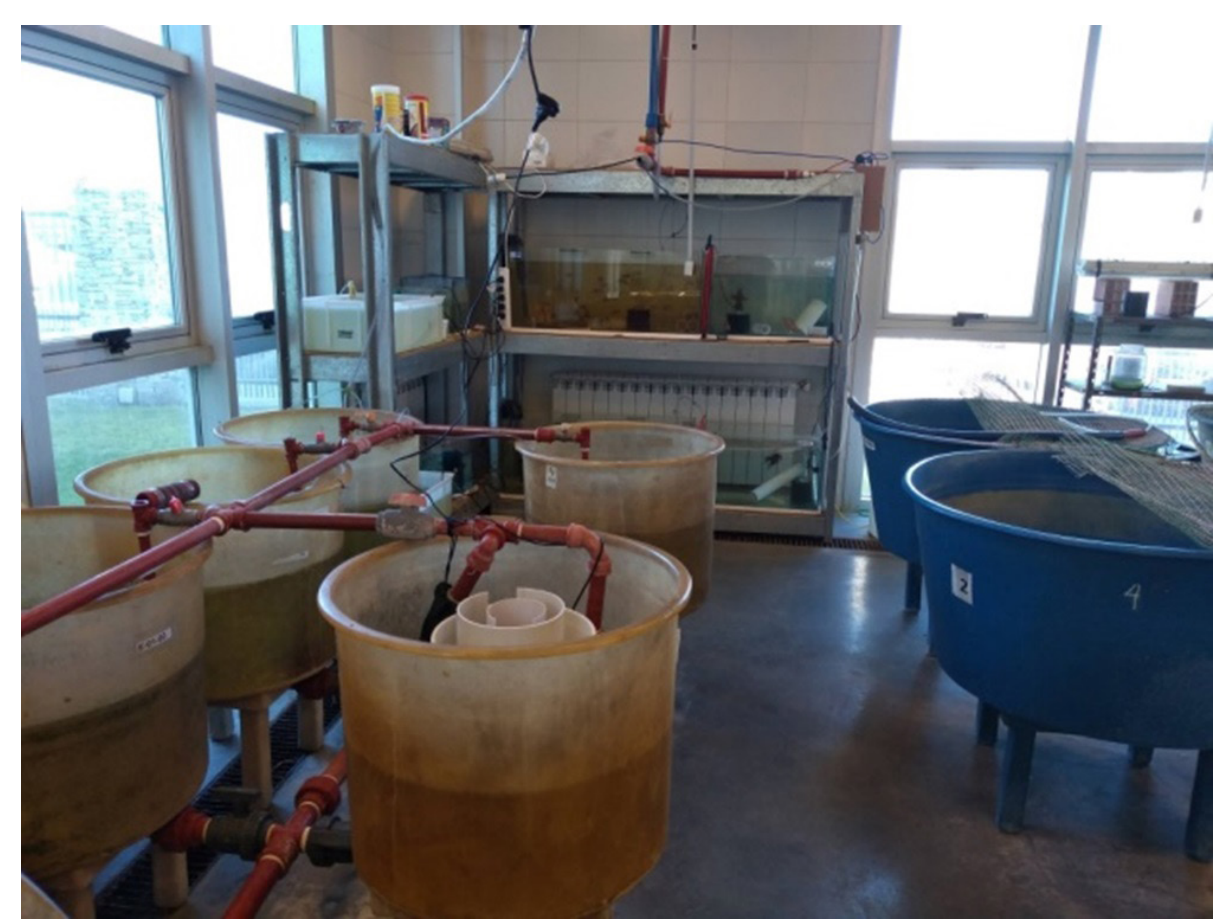

Figura 1. Sector del Laboratorio destinado a la cría de peces ornamentales

Se utilizó un Sistema de Recirculación de Agua (SRA) con tratamientos unitarios de su calidad (Figura 2). El SRA se conformó con 5 tanques circulares de $48 \mathrm{~cm}$ de altura y $60 \mathrm{~cm}$ de diámetro de plástico reforzado con fibra de vidrio, un tanque de iguales características para contener la bomba sumergible (HSB1500) y el filtro biológico; un filtro sedimentador, construido con un balde plástico de 20 litros, de altura $38 \mathrm{~cm}$ y de $28 \mathrm{~cm}$ de diámetro, dos piezas interiores de caños de PVC seccionadas de $18 \mathrm{~cm}$ de largo y $10 \mathrm{~cm}$ de diámetro; la entrada de agua inferior y la salida del agua superior, el cual se observa en la Figura 3. La filtración biológica se realizó con un filtro tipo semisumergido y para su diseño se utilizaron los cálculos generales de Álvarez y Quevedo (2006), modelándose el sistema de la siguiente forma:

- Carga máxima a utilizar: $500 \mathrm{~g}$.

- Tasa de alimentación máxima a utilizar: $5 \%$ de la biomasa total, resultando una cantidad de alimento diario máximo de $25 \mathrm{~g}$.

A través de estos cálculos se estimó para el biofiltro un área de 0,613 m2 para una producción diaria de nitrógeno amoniacal total (NAT) establecida en 0,92 g NAT/día. Cada unidad de sustrato de $2,5 \mathrm{~cm}$ de manguera corrugada proporcionó $0,0024 \mathrm{~m} 2$, por lo cual fueron necesarias 256 unidades para completar la superficie requerida. El sistema de aireación consistió de una bomba de aire (blower) que comprime aire atmosférico y lo inyecta a los tanques de cultivo a través de mangueras cristal de $1 / 8$ pulgada con difusores de aire. El acondicionamiento térmico del sistema se llevó a cabo a través de la calefacción proporcionada por radiadores ubicados en el ambiente general del laboratorio, y el uso de un termostato eléctrico para mantener la temperatura adecuada, siendo el rango ideal de $H$. bimaculatus de entre $22-28^{\circ} \mathrm{C}$ (Jiménez-Alvarado, 2007).

El agua utilizada proveniente de la red de agua potable local fue tratada inicialmente por 
ósmosis inversa para obtener agua blanda (0-50 $\left.\mathrm{mg} \mathrm{CO}_{3} \mathrm{Ca} / \mathrm{litro}\right)$, requerida por la especie, utilizándose para ello un equipo Marca Aquatic Life Ro Buddle, provisto de un cartucho de sedimentación 330249 y un cartucho de carbono 330250; el mismo fue conectado a una bomba Booster Pump WE-P6010. El total de agua del sistema fue 471 litros y el caudal 75 litros/h; la recirculación del sistema, cada 1 h $24^{\prime}$. La conducción del agua del SRA se realizó con tubos de polipropileno de $3 / 4$ pulgadas de diámetro, con llaves esféricas para la entrada y la salida del agua de cada tanque de cría.

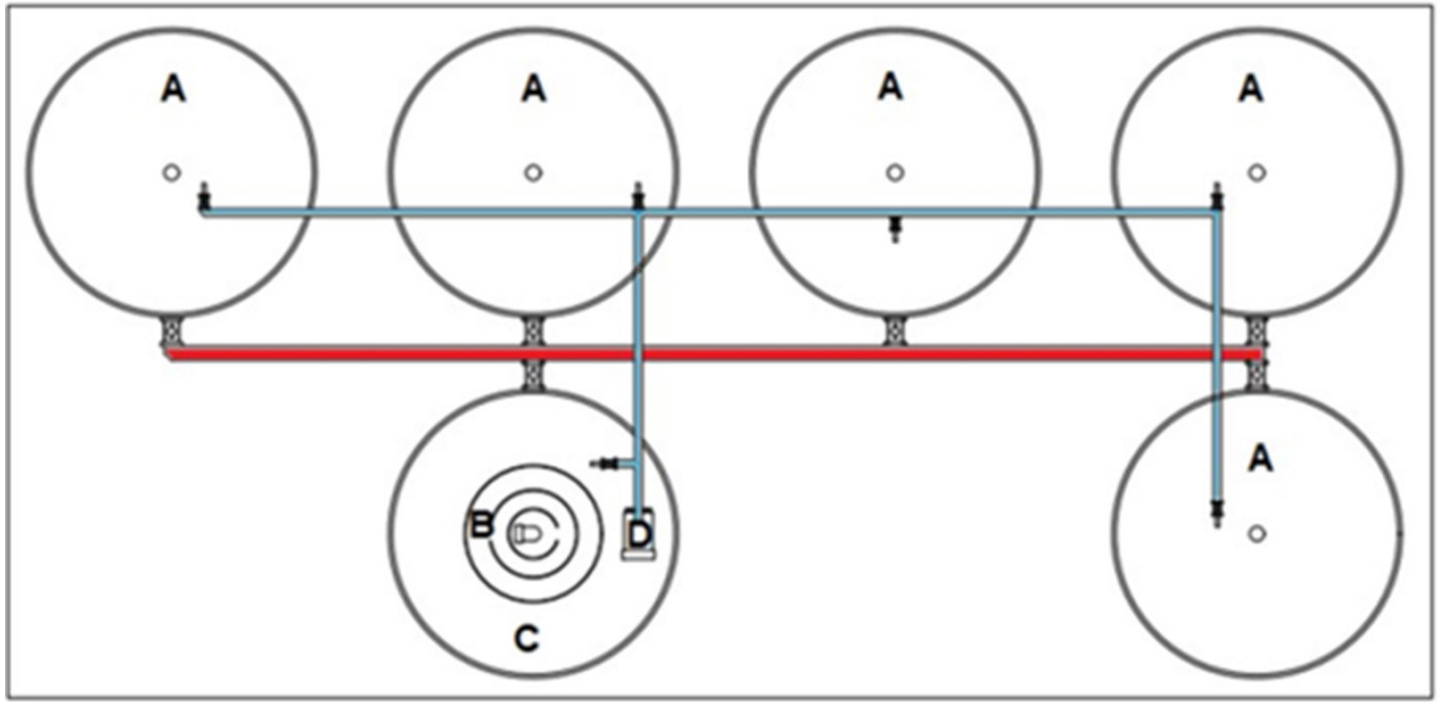

Figura 2. Vista en planta del Sistema de Recirculación de Agua (SRA) experimental: A) tanques de cultivo; B) filtro sedimentador; C) tanque contenedor del filtro biológico y de la bomba sumergible; D) bomba sumergible. Sistema de conducción de agua: tratada y efluente.

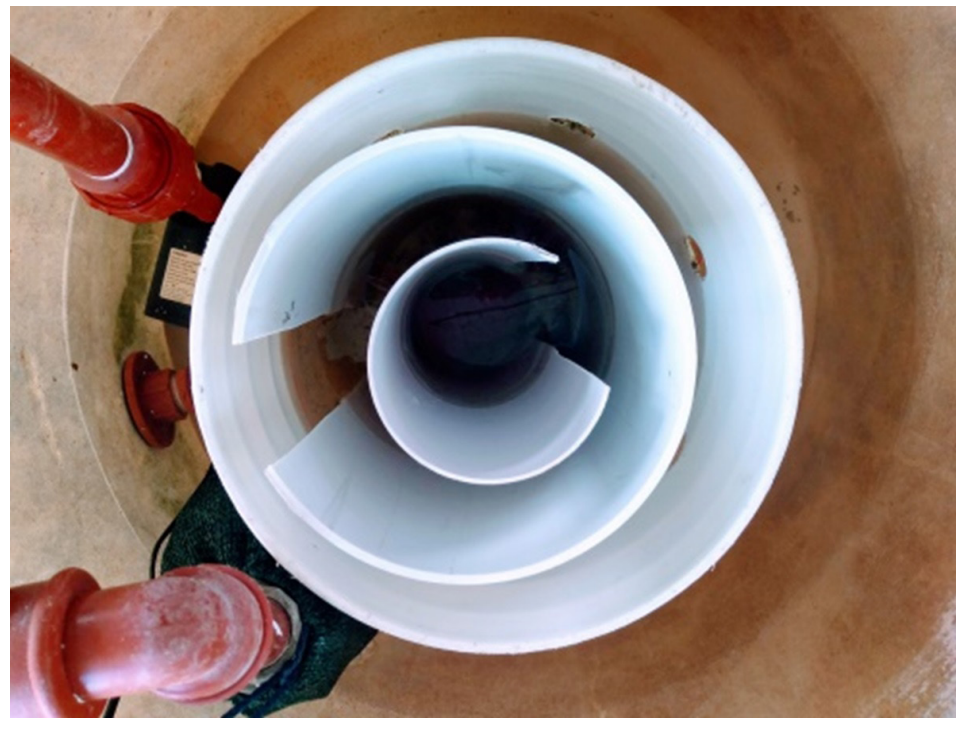

Figura 3. Vista superior del filtro sedimentador del sistema de cultivo 
Los peces juveniles tuvieron inicialmente un peso promedio $(\mathrm{Pp})$ de $0,2663 \pm 0,1069 \mathrm{~g}$ y

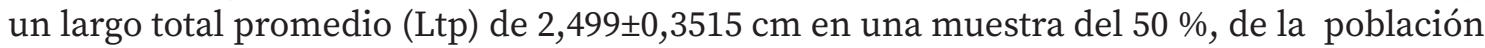
total de 200 ejemplares, distribuidos en cuatro tanques de cría, 50 individuos en cada uno en forma aleatoria y con una densidad adecuada para la especie. Se aplicaron dos tratamientos de alimentación (T), por duplicado: T1 control, un alimento comercial con 47,5 \% proteína bruta y 6,7 de lípidos (Marca Tetra Tropical R), y T2 alimento experimental, con la incorporación de harinas de residuos de langostino, de cebada y complementos de algas, la cual fue preparada en el laboratorio.

Los análisis de la composición de las harinas de residuos de cabeza de langostino y de bagazo de cebada, fueron realizados por el Laboratorio de Análisis Industriales de la FRMdP, según AOAC (1997). Los resultados de su composición y los métodos utilizados se observan en la Tabla I.

\begin{tabular}{|l|c|c|c|}
\hline & C & L & Métodos \\
\hline Determinación & \multicolumn{2}{|c|}{ Resultados } & Secado en estufa de aire \\
\hline Humedad \% & 10,1 & 4,9 & Calcinación en mufla \\
\hline Cenizas \% & 3,0 & 17,0 & \begin{tabular}{c} 
SER 148 \\
\hline Grasas totales \%
\end{tabular} \\
\hline Proteínas \% & 16,6 & 11,0 & Kjeldahl \\
\hline Carbohidratos \% & 65,4 & 9,0 & Cálculo \\
\hline Valor energético kcal/100 g & 370,6 & 367,4 & Cálculo \\
\hline Fibra bruta \% & 8,8 & 9,4 & Digestión ácida y alcalina \\
\hline
\end{tabular}

Tabla I. Composición de harinas de residuos de bagazo de cebada (C) y de cabeza de langostino (L), y métodos utilizados en cada determinación.

Otras harinas, como la de pescado y de soja utilizadas, de composición conocida, aportaron 58,0 y 35,2 \% de proteínas, respectivamente. Así, se calculó en forma teórica el porcentaje de inclusión de las mismas en el alimento T2 para un aporte de $40 \%$ de proteínas (Tabla II).

\begin{tabular}{|c|c|c|c|}
\hline Harinas & $\begin{array}{c}\text { Cantidad } \\
\%\end{array}$ & $\begin{array}{c}\text { Cantidad } \\
\text { g/kg }\end{array}$ & $\begin{array}{c}\text { Proteína } \\
\%\end{array}$ \\
\hline harina de pescado & 15 & 250 & 58,0 \\
\hline harina de langostino & 15 & 250 & 58,1 \\
\hline harina de cebada & 15 & 150 & 16,4 \\
\hline harina de soja & 5 & 142 & 35,2 \\
\hline
\end{tabular}

Tabla II. Principales harinas utilizadas, cantidad (\% y g/kg) y aporte de proteínas (\%) utilizadas en el alimento experimental (T2). 
Se incorporó almidón de maíz 3,5 \%, aceite de girasol 6\%, harinas comerciales de las algas Espirulina 1,5 \% y de U. pinnatifida 1,5\%, solución de vitaminas y minerales $1 \%$, Vitamina C $0,1 \%$, y $400 \mathrm{ml}$ de agua. Obtenida una mezcla homogénea se elaboraron los pellets mediante el uso de un molino para carne, los cuales fueron extendidos en bandejas metálicas y secados en una estufa a $45^{\circ} \mathrm{C}$ durante 24 horas. El tamaño de los pellets fue ajustado al tamaño de la boca de los peces, para luego almacenarse en el refrigerador a $5{ }^{\circ} \mathrm{C}$. La composición general de los alimentos (T1 y T 2) se observa en la Tabla III. Los principales componentes del alimento comercial fueron aportados por la información del producto comercial, y del experimental T2 por análisis en el Laboratorio de Análisis Industriales de la FRMdP, de acuerdo a la misma metodología ya consignada en la Tabla I.

\begin{tabular}{|l|c|c|}
\hline \multirow{2}{*}{ Componente \% } & Alimento comercial & Alimento experimental \\
\hline Proteínas & 47,5 & 38,5 \\
\hline Lípidos & 6,5 & 13,9 \\
\hline Fibra & 2,0 & 4,9 \\
\hline Humedad & 6,0 & 6,8 \\
\hline
\end{tabular}

Tabla III. Composición química general de los alimentos comercial y experimental

La cantidad de alimento ofrecida diariamente se calculó en base a la biomasa de los peces, con un nivel de alimentación de $5 \%$ de peso vivo/día, en dos raciones diarias. Se alimentó 6 días/semana. El alimento se fue ajustando a través de los correspondientes muestreos. Los mismos se realizaron cada 15 días durante tres meses, registrándose el peso $\mathrm{P}(\mathrm{g})$ y el largo total Lt $(\mathrm{cm})$ del $50 \%$ de los ejemplares de cada tanque de cultivo, y del $100 \%$ al finalizar la experiencia. Se calcularon los parámetros de crecimiento de acuerdo a lo propuesto por Wootton (1991), a partir de los valores de P promedio inicial y final: Crecimiento absoluto (g), Tasa de crecimiento absoluto (g), Crecimiento relativo (g), Tasa de crecimiento relativo (g) y Tasa de crecimiento específica (mg/día). Además, la biomasa total y la supervivencia, para ambos tratamientos. Se observó el aspecto externo de los peces y su comportamiento a través del tiempo. Todos los individuos fueron sometidos a un ayuno de $24 \mathrm{hs}$ antes de cada biometría.

Se registraron los parámetros de calidad de agua durante toda la experiencia; el pH y la temperatura del agua de los tanques del SRA se determinaron mediante un equipo Phmetro marca "Adwa" AD12; el oxígeno disuelto mediante un equipo marca Aqua COMBO HM370 y los compuestos nitrogenados amonio, nitrito y nitrato y la dureza total mediante el uso de kits de acuario Marca Tetra. La concentración de NAT, nitritos y nitratos se mantuvo dentro de los rangos 0,01-1,5; 0-0,3; 0-100 (mg/l), respectivamente. La temperatura, dureza, $\mathrm{pH}$ y oxígeno disuelto registrados durante la experiencia fueron: $24-26{ }^{\circ} \mathrm{C}$; 0-100 $\mathrm{mg} \mathrm{CO} 3 \mathrm{Ca} / \mathrm{litro}$; 7-8,5 unidades de $\mathrm{pH}$, y 5-6 mg/litro, respectivamente. La temperatura se midió tres veces y los demás parámetros una vez, por semana. La concentración de nitratos indicó el reemplazo parcial de agua de acuerdo a los valores recomendados para peces ornamentales, $\leq 100$ $\mathrm{mg} /$ litro. Se realizó sifoneo del fondo de los tanques de cultivo dos veces por semana y del filtro sedimentador cuando fue necesario.

Los análisis estadísticos realizados consistieron en la descripción univariada y bivariada 
de las variables observadas y, en una segunda etapa, en la aplicación de metodología de inferencia pertinente. La base de datos constó de 800 observaciones obtenidas a partir de los muestreos realizados durante la experiencia de cultivo. Las variables observadas fueron $\mathrm{P}(\mathrm{g})$, Lt (cm) y Tratamiento (T1 y T2). Del análisis univariado se obtuvieron las medidas estadísticas de cada variable y se aplicó la prueba de normalidad para P y Lt. Se aplicó la prueba $\mathrm{F}$ para la comparación de varianzas de las variables observadas respecto a los dos tratamientos. Para analizar la distribución de las variables respecto a los dos tratamientos se aplicó metodología no paramétrica (Prueba Mann-Whitney). Para medir el grado de asociación lineal entre las variables $\mathrm{P}$ y Lt se calculó el índice de correlación de Spearman. El nivel de significación utilizado en todas las pruebas fue del $5 \%$. Todo el análisis estadístico se desarrolló por medio del soft estadístico R-Project. $\mathrm{R}$ es un lenguaje de programación y un entorno de software libre para computación estadística y gráficos respaldados por la Fundación R para Computación Estadística. Desarrollador: R Core Team; Versión 4.0.4 (https://www.r-project.org/).

\section{Resultados}

En la primera etapa del análisis univariado se consideraron las observaciones de la variable $\mathrm{P}$ obtenidas del primer muestreo; la distribución de $\mathrm{P}$ no presentó distribución normal. Aplicando la prueba para la comparación de distribuciones (o medianas) de P respecto a los dos grupos de ejemplares, los resultados evidenciaron que las mismas no difieren significativamente antes de la aplicación de los dos tratamientos (T1, T2).

$\mathrm{Al}$ considerar todas las observaciones realizadas dentro del período de estudio a través de los muestreos, se observó que los parámetros de crecimiento tanto para $\mathrm{P}$ como para Lt no presentaron distribución normal, por lo cual se utilizó metodología no paramétrica.

El coeficiente de correlación de Spearman entre ambas variables fue 0,9818, lo cual indica una elevada relación lineal entre las mismas.

En cuanto al análisis del efecto de los dos tratamientos sobre P y Lt, se evidenció que las distribuciones de ambas variables presentaron diferencias significativas $(p<0,05)$. Es decir, el efecto tratamiento incidió en el comportamiento de las dos variables analizadas. La prueba de comparación de varianzas respecto a los dos tratamientos, también mostró diferencias significativas $(\mathrm{p}=0,00)$.

Al no presentar distribución normal, las medianas son las medidas estadísticas más representativas cuando hay asimetría de las variables. En la Figura 4 se observan las distribuciones de las variables P y Lt para T1 y T2 al finalizar el presente estudio, representadas mediante diagramas de caja. Los valores de las medianas obtenidos fueron, de P 1,225 en T1 y 0,885 en T2, y de Lt 3,9 en T1 y 3,5 en T2. Cada caja contiene el $50 \%$ de los valores de cada variable para cada tratamiento. Las líneas o bigotes que salen de las cajas identifican los valores mínimo y máximo de cada variable para casos extremos leves; por fuera se pueden reconocer los valores atípicos o extremos, representados por círculos, hallándose dos en $\mathrm{P}$ T2, valor de 3,05 (observación 570) y 3,36 (observación 591). Se muestra una gran dispersión, dada por la extensión de las líneas o bigotes hacia arriba y hacia abajo. 


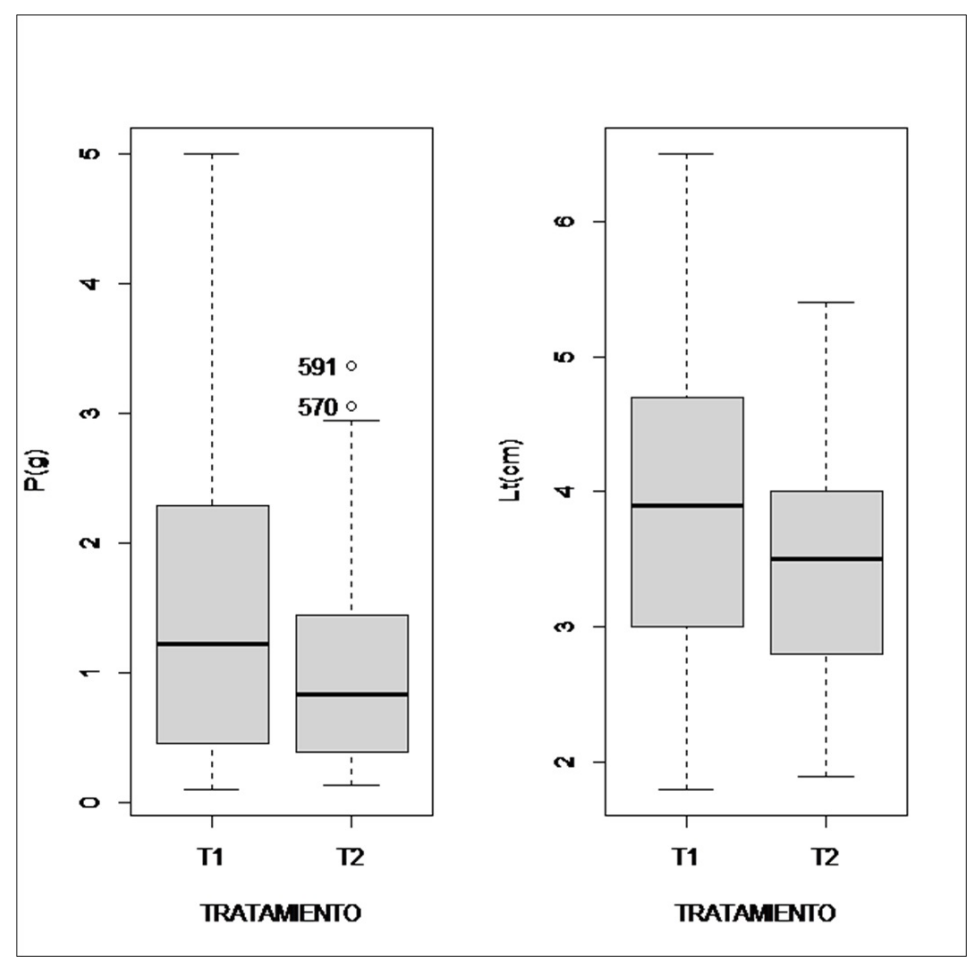

Figura 4. Distribución de Peso P $(\mathrm{g})$ y Largo total Lt $(\mathrm{cm})$ correspondiente al tratamiento control (T1) y al tratamiento experimental (T2) al finalizar la experiencia de crecimiento de juveniles de $H$. bimaculatus, mediante diagramas de caja (medianas: líneas negras dentro de cada caja; dispersión: dada por la extensión de las líneas cortadas; valores atípicos: observaciones 570 y 591 en P T2).

En la Tabla IV se observan los datos técnicos correspondientes al inicio y final del presente trabajo experimental.

\begin{tabular}{|c|c|c|c|c|c|c|}
\hline \multirow{2}{*}{ Parámetro } & \multicolumn{3}{|c|}{ Inicio } & \multicolumn{3}{c|}{ Final } \\
\cline { 2 - 7 } & $\mathrm{T} 1$ & & $\mathrm{~T} 2$ & $\mathrm{~T} 1$ & & $\mathrm{~T} 2$ \\
\hline $\mathrm{N}^{\circ}$ de peces & 100 & & 100 & 100 & & 96 \\
\hline Pp (g) & $0,2598 \pm 0,098$ & & $0,2728 \pm 0,16$ & $2,9115 \pm 0,81$ & & $1,65625 \pm 0,56$ \\
\hline Ltp (cm) & $2,504 \pm 0,33$ & & $2,494 \pm 0,37$ & $4,6600 \pm 0,73$ & & $4,1625 \pm 0,54$ \\
\hline Biomasa total (g) & 25,98 & & 27,28 & 291,15 & & 159,00 \\
\hline Densidad (litros/pez) & & 1,57 & & & - & \\
\hline Sobrevivencia (\%) & & - & & 100 & & 98 \\
\hline
\end{tabular}

Tabla IV. Parámetros técnicos correspondientes a los tratamientos T1 y T2 al inicio y final de la experiencia de crecimiento de juveniles de $H$. bimaculatus: $\mathrm{N}^{\circ}$ de peces, $\mathrm{P}$ promedio $\mathrm{Pp}(\mathrm{g})$, Lt promedio Ltp (cm), Biomasa total (g), densidad inicial y Sobrevivencia (\%).

Los resultados de los parámetros de crecimiento Pp y Ltp del tratamiento T2 fueron positivos, si bien no alcanzaron los valores correspondientes a T1; la sobrevivencia fue muy 
elevada, 98 \%. En la Tabla V se observan los parámetros de crecimiento en ambos tratamientos, siendo el valor de la tasa de crecimiento específica de $2 \mathrm{mg}$ /día en T2, mientras que en T1 fue de 2,69 mg/día.

\begin{tabular}{|l|c|c|}
\hline Parámetros & T1 & T2 \\
\hline Peso inicial promedio $(\mathrm{g})$ & 0,2598 & 0,2728 \\
\hline Peso final promedio $(\mathrm{g})$ & 2,9115 & 1,6563 \\
\hline Crecimiento absoluto $(\mathrm{g})$ & 2,6517 & 1,3835 \\
\hline Tasa de crecimiento absoluto $(\mathrm{g})$ & 0,0295 & 0,0154 \\
\hline Crecimiento relativo $(\mathrm{g})$ & 10,2100 & $\mathbf{5 , 0 7 0 0}$ \\
\hline Tasa de crecimiento relativo (g) & 0,1100 & 0,0560 \\
\hline Tasa de crecimiento específica (mg/día) & 2,69 & 2,00 \\
\hline
\end{tabular}

Tabla V. Parámetros de crecimiento de los juveniles de H. bimaculatus en ambos tratamientos, T1 y T2.

Los ejemplares se comportaron como cardumen durante todo el tiempo de estudio, no observándose agresión entre los peces en cada tanque de cultivo.

En cuanto al aspecto externo, la coloración característica rojiza fue más intensa en ejemplares alimentados con la dieta comercial, T1, en comparación con los correspondientes a T2, color rosa claro (Figura $5 \mathrm{~A}$ y B).
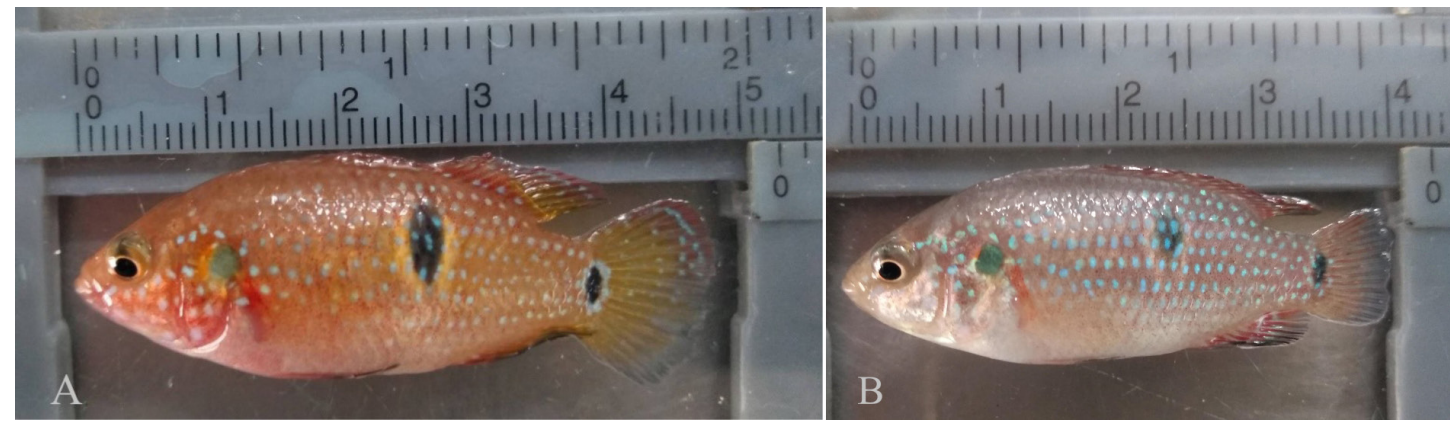

Figura 5. Ejemplares juveniles de H. bimaculatus obtenidos al finalizar el estudio, con la coloración rojiza característica de la especie en el tratamiento T1 (A), y con una coloración rosada en el tratamiento T2 (B).

Los valores de los parámetros físico-químicos del agua fueron adecuados durante la cría de $H$. bimaculatus en el SRA. El agua agregada debido a la pérdida por evaporación y limpieza por sifoneo de los tanques contenedores y del filtro sedimentador fue 2,5\% del volumen total/día. El reemplazo de agua total del SRA fue del $10 \%$ una sola vez, cuando los nitratos alcanzaron valores $\geq 100 \mathrm{mg} /$ litro.

\section{Discusión y conclusiones}

Los SRA en Acuicultura permiten mantener el mismo volumen de agua en constante circulación para metabolizar los desechos a través de sistemas de filtración, siendo sistemas semicerrados, es decir, que no poseen interacciones significativas con el ambiente (Losordo, 1998; Piedrahita, 2003; Timmons y Ebeling, 2007). El crecimiento de los peces es mayor que en los 
sistemas tradicionales, debido a la mejora en las condiciones de calidad de agua, disponibilidad de alimento y reducción de pérdidas energéticas durante la búsqueda del mismo (Lasur y Britt, 1997; Schuster y Stelz, 1998), además de permitir conocer el número exacto y estado de desarrollo de los peces, calcular la biomasa exacta a lo largo del ciclo productivo y racionar el alimento, como facilitar la detección de signos de enfermedad (Timmons y Losordo, 1994). En el presente trabajo, el SRA utilizado para el cultivo de juveniles de $H$. bimaculatus permitió trabajar con un número de animales representativo y con dos tratamientos de alimentación y réplicas, manteniendo las mismas condiciones de los parámetros de calidad de agua adecuadas para la especie en cada unidad contenedora, lo cual es muy importante en el trabajo experimental. Este sistema se podría aplicar a otras especies ornamentales, de similares características, teniendo en consideración la capacidad del sistema, su comportamiento y el espacio-volumen requerido.

Con respecto al alimento, si bien las proteínas son los nutrientes más importantes que afectan el rendimiento productivo de los peces, son a su vez uno de los componentes más costosos en la dieta (Gutiérrez et al, 2009); su requerimiento en el alimento es alto y varía entre 35-56 \% (Jauncey y Ross, 1982), siendo la harina de pescado la fuente principal de su provisión. En el presente trabajo el suministro de la dieta T2 obtuvo buenos resultados aunque los parámetros de crecimiento no alcanzaron a los de la dieta control (T1). La tasa específica de crecimiento es afectada por los tipos de alimentos proporcionados a los organismos y la cantidad de proteínas, y es un indicador de la calidad de las mismas en las dietas, y en condiciones controladas la ganancia en peso de los peces está en proporción a los aminoácidos esenciales suministrados (Tacon, 1987). Si bien en estudios con otras especies de la familia Cichlidae se han logrado buenos resultados utilizando 32-34 \% de proteína bruta (Ribeiro et al, 2008; Zuanon et al, 2009), en el presente trabajo el aporte de proteínas en el tratamiento alternativo no fue suficiente. La sobrevivencia fue muy elevada al comparar ambos tratamientos. En la coloración de los juveniles se observó en general una deficiencia de pigmentación en T2. El pigmento astaxantina es un producto estandarizado y químicamente estable con una concentración óptima en los alimentos comerciales para peces de ornato que representa un extra de 10-15\% del costo del alimento (Kop y Durmaz, 2008), por lo cual para valorizar el aspecto externo de los mismos se deberá tener en cuenta incorporar otras fuentes naturales de pigmentos carotenoides y realizar la determinación analítica en el alimento.

Las harinas de cabeza del langostino y de bagazo de cebada son materias primas alternativas sustentables que pueden ser consideradas para suplantar parcialmente a la harina de pescado.

Los SRA son tecnologías amigables con el ambiente. Se han implementado en un número reducido de especies ornamentales y en bajas densidades, debiéndose adecuar las características de dichos sistemas de cultivo intensivo con los requerimientos propios de los peces. Por otro lado, en los SRA se puede utilizar agua de lluvia y energía generada en paneles solares o hélices eólicas (White et al, 2004), como alternativas en la implementación de procesos sustentables. Los SRA están siendo muy utilizados en la producción de peces para consumo, pero debido a las altas densidades de cultivo con las que se trabaja éstos resultan bastante complejos. De otra manera, resulta más sencilla la implementación de los SRA para la producción de peces ornamentales con sus consiguientes ventajas, tanto ambientales como económicas.

\section{Agradecimientos}

El presente trabajo fue posible gracias a los fondos provistos por la Universidad Tecnológica Nacional, en el marco del PID MSUTNMP5000. 


\section{Referencias}

Álvarez, M., y Quevedo, J. (2006). “Diseño y evaluación de un sistema de recirculación con fines didácticos y experimentales para la acuicultura”. Revista de la Academia Canaria de Ciencias, 17(4): 57-72.

AOAC (1997). Official Methods of Analysis. 16th ed. Vol. II. Association of Official Analytical Chemists International, Maryland, USA.

Argento, F., Sempere, C. y van Lierde, F. (2016). Factibilidad técnica y económica de la producción de Espirulina. Proyecto final de Ingeniería Industrial- ITBA, 81pp.https:// ri.itba.edu.ar/bitstream/handle/123456789/858/Factibilidad\%20t\%C3\%A9cnica\%20 y\%20econ\%C3\%B3mica\%20de\%20la\%20producci\%C3\%B3n\%20de\%20\%20spirulina. pdf? sequence $=1 \&$ isAllowed $=y$

Casas, G., Gil M., Commendatore, M. y Torres, A. (2010). "Evaluación de la composición químico-nutricional y del nivel de metales pesados e hidrocarburos en Undaria pinnatifida de los golfos San José y Nuevo, Chubut”. Informe Final. Proyecto financiado por la Secretaría de Ciencia, Tecnología e Innovación, Prov. de Chubut. Cenpat-Conicet, Puerto Madryn, Chubut, $47 \mathrm{pp}$.

Cira, L.A., Huerta, S., y Shirai, K. (2002). "Fermentación láctica de cabezas de camarón (Penaeus sp.) en un reactor de fermentación sólida”. Revista Mexicana de Ingeniería Química, 1: 45-48.

Ferrari, J.L., Villagra, S., Claps, L. y Tittonel, P. (2017). "Reutilización de bagazo de cebada cervecera por secado y pelletización como suplemento forrajero”. Revista Presencia, 67: 4346.

Fox, C.J., Blow, P., Brown, J.H. y Watson, I. (1994). “The effect of various processing methods on the physical and biochemical properties of shrimp head meals and their utilization by juvenile Penaeus monodon Fab”, Aquaculture, 122: 209-226.

Gutiérrez A, F.W., Zaldivar, R, J. y Contreras S, G. (2009). "Efecto de varios niveles de energía digestible y proteína en la dieta sobre el crecimiento de gamitana (Colossoma macropomum) CUVIER 1818”. Revista de Investigaciones Veterinarias del Perú, 20(2): 178-186.

Hepher, B. (1993). Nutrición de peces comerciales en estanques. México, Limusa S.A.

Hertrampf, J.W. y Piedad-Pascual, F. (2000). Handbook on ingredients for aquaculture feeds, Netherlands, Kluwer Academia Publishers, 365-371.

Honorato, G.C., Olveira, E.L., de S. Alsina, L.S. y Magalhaes, M.M.A. (2006). "Estudio del proceso cinético del secado de cefalotórax de camarón”. Información Tecnológica, 16(4):310.

Jiménez-Alvarado, D.L. (2007). "Efecto del sexo del cíclido joya (Hemichromis bimaculatus) (Pisces: Cichlidae) ante los cambios de temperatura”. Anales Universitarios de Etologia, 1:53-57.

Jauncey, K. y Ross, B. (1982). A guide to Tilapia Feeds and Feeding. Stirling (UK): Institute of Aquaculture, University of Stirling.

Kop, A. y Durmaz, Y. (2008). "The effect of synthetic and natural pigments on the colour of the cichlids (Cichlasoma severum sp., Heckel 1840)”. Aquaculture International, 16:117-122. 
Lam Romero, F., Barroso Sandoval, V. y Domínguez Brito, J. (2012). "Evaluación de tres tipos de alimento en el crecimiento preliminar de la "Cachama Blanca" (Piaractus brachypomus) en la localidad de Santa Clara, Provincia de Pastaza, Ecuador". Revista Amazónica de Ciencia y Tecnología, 4:137.

Lasur, A. y Britt, C. (1997). "Pond recirculating production systems", Southern Regional Aquaculture Center, SRAC, 455:1-7.

Lladser, N.L., Becerra, A.A., Vargas, A., Andrea, A., Rost, E., Crettón, M., Mazzuca, M. y Sobczuk, T. (2014). "Propuesta de des-primarización en el procesamiento del langostino del Golfo San Jorge”, VII Congreso Argentino de Ingeniería Industrial (COINI). 30 y 31 de octubre 2014. Puerto Madryn, Chubut.

Losordo, T.M. (1998). "Recirculating Aquaculture Production Systems: The Status and Future”. Aquaculture Magazine, 24(1):38-45.

Piedrahita, R. (2003). "Reducing the potential environmental impact of tank aquaculture effluents through intensification and recirculation”. Aquaculture, 226: 35-44.

Ribeiro, F.A.S., Preto, B.L. y Fernandes, J.B.K. (2008). "Sistemas de criação para o acarábandeira (Pterophyllum scalare)”. Acta Scientiarum. Animal sciences, 30(4): 459-466.

Schuster, C. y Stelz, H. (1998). "Reduction in the make-up water in semiclosed recirculating aquaculture systems”. Aquaculture Engineering, 17:167-174.

Shoemaker, R. y Richards-Rajaduraí, N. (1991). “Shrimp waste utilización”. Infofish Technical Handbook, Kuala Lumpur, 4:20.

Tacon, A. (1987). The nutrition and feeding of farm fish and shrimp - a training manual- I. The essential nutrients. FAO. Trust Fund GCP/RLA/075/ITA. Brasilia, Brasil.

Timmons, M. y Losordo, M. (1994). Aquaculture water reuse systems: engineering design and management. Amsterdam; New York: Elsevier.

Timmons, M. y Ebeling, J. (2007). Recirculating Aquaculture. Northeastern Regional Aquaculture Center (NRAC), Michigan State University, Lansing, Publication núm. 01-007.

Vásquez-Torres, W., Pereira-Filho, M. y Arias-Castellanos, J.A. (2011). "Optimum dietary crude protein requirement for juvenile cachama Piaractus brachypomus". Ciência Rural, 41(12):2183-2189.

White, K., O’Neill, B. y Tzankova, Z. (2004). “At a Crossroads: Will Aquaculture Fulfill the Promise of the Blue Revolution?” http://mauricoast.com/pics/mauricoastpics/reports_ crossroads.pdf

Wootton, R. F. (1991). Ecology of Teleost Fishes. Fish and Fisheries. Series Y. Chapman \& Hall, 2-6 Bodanz Row, London SE 1 8HN.

Zuanon, J.A.S., Salaro, A.L., Silveira, S.S.M., de Oliveira, L.M.A., Balbino, E.M. y Siqueira, E.A. (2009). "Dietary Protein and energy requirements of juvenile freshwater angelfish". Revista Brasileira de Zootecnia, 38(6):989-993. 